\title{
Numerical Study on the Interface Evolution of the Unsteady Supercavity Flows with a Strong Gas Jet
}

\author{
Min Xiang*, Xiaoyu Zhao, Zeyang Xie \\ College of Aerospace Science and Engineering, National University of Defense Technology, \\ Changsha 410073, P.R. China \\ * Corresponding Author Email: xiangmin333@ hotmail.com
}

\begin{abstract}
Artificial supercavition is one of the most prospective technique for underwater drag reduction, but it still faces some unsolved roadblocks, like cavity stability, noise, power etc. Jet propulsion could provide enough thrust for underwater vehicle. However, it could simultaneously shrink effective cavity volume, and cause cavity interface deform and fluctuate. This article aims at investigating the instability of cavity with the strong jet impingement, and analyzing jet behavior in restricted space. Besides jet intensity, the distance between nozzle exit and cavity closure is considered as a very important parameter in jet/supercavity interaction. As the relative position varies, it will lead to diverse cavity surface evolution and different jet entrainment effect in cavity. In this paper, a multiphase model using coupled VOF and level set method is adopted to capture gas-liquid interface. Simulation for a supercavitating case without jet will be firstly carried out under different ventilation flow rate and comparison will be carried out with the experimental data to validate the numerical model. Then, by changing the position of jet nozzle exit, a series of numerical simulations of supercavitation flows is performed. A non-dimensional distance parameter is proposed to define the interaction schemes between jet and cavity.
\end{abstract}

Keywords: Jet, Supercavity, Instability, Relative position, CLSVOF.

\section{Introduction}

Cavitation is a dynamic phenomenon that occurs when underwater vehicles travel with such a high speed that the pressure around the vehicle surface decrease to the vapor saturation pressure of the liquid. High drag reduction could be achieved when the vehicle is entirely covered by the cavity which is defined as supercavitation. However it is difficult to achieve supercavition under natural conditions. Reichardt(1946) firstly used the method of artificial air injection to form a steady cavity that covered the entire surface of an object. That pioneering work provided the possibility for engineering application of drag reduction technology by cavitation. Afterwards an amount of works that contain theoretical and experimental work on ventilated cavitation were performed by Garabedian(1956), Cox and Clayden(1956), Waid(1957), Campbell and Hilborne(1958), Logvinovich(1969), Epshtein(1973), Semenenko(2001), and so on. The empirical formulas of the steady cavity shape and ventilation demand for supercavity formation without external perturbations have been built. In one word, ventilated cavity flows without jet depend on several non-dimensional fundamental parameter: cavitation number, $\sigma_{c}$, Froude number, Fr, the gas ventilation coefficient, $\mathrm{Cq}$.

$$
\begin{gathered}
\sigma_{c}=\frac{2\left(p_{\infty}-p_{c}\right)}{\rho_{\infty} U_{\infty}^{2}} \\
F r=\frac{U_{\infty}}{\sqrt{g D_{n}}} \\
C_{\mathrm{q}}=\frac{Q_{D}}{\rho U_{\infty} D_{n}^{2}}
\end{gathered}
$$

In those relation, $p_{\infty}, \rho_{\infty}$ and $U_{\infty}$ denote the free liquid flow pressure, the liquid density and velocity respectively; $p_{c}$ is the pressure inside the supercavity; $D n$ is the cavitator diameter; and $Q_{D}$ refers to the gas ventilation mass flow rate.

Ensure the stability of the cavity shape is the premise of drag reduction by supercavitation technology, however the cavitation stability is disturbed by many factors in fact. Recently, the effects of the gas jet from exhaust nozzle on an established steady cavity has got some attention. Paryshev(2006) gave an approximate mathematical models of cavity when it closes on a central liquid or incompressible gas jet. According to his theory, the effect of jet on the cavity was governed by two non-dimensional numbers: $\bar{P}$, the ratio of the stagnation pressure of the surrounding flow to that of the jet, and $\bar{J}$, the 
ratio of the jet momentum flux to cavitator drag, which are defined as follows:

$$
\begin{aligned}
& \bar{P}=\frac{\rho_{\infty} U_{\infty}^{2}}{\rho_{j e t} U_{j e t}^{2}} \\
& \bar{J}=\frac{\dot{m}_{j e t} U_{j e t}}{W_{c}}
\end{aligned}
$$

Where $U_{j e t}$ denotes the mean jet velocity at the nozzle exit; $\dot{m}_{\text {jet }}$ denotes the mass flow_rate of the jet; $W_{c}$ is the cavitator drag. Jet/supercavity interaction is divided into three categories: (1) when $\bar{P}>1$ and $\bar{J}<1 / 2$, the jet is totally obstructed by the cavity, and returns into it. (2) when $\bar{P}>1$ and $\bar{J}>1 / 2$, the jet is divided into two parts, a portion goes out of the cavity while the other portion returns and inflates the cavity. (3)when $\bar{P}<1$, the jet isn't completely obstructed and leaves the cavity. This moment the cavity closes on the central jet in the form of the Efros-type closure. Moeny(2015) conducted a series of experiments on jet-supercavity interaction in the $1.22 \mathrm{~m}$ dia. Garfield Thomas Water Tunnel. By changing the jet mass flow rates from 0 to $0.11 \mathrm{~kg} / \mathrm{s}$, experiments were performed at two tunnel speeds and for two nozzle diameters. Collected data well supported Paryshev's theory models. Kinzel et al(2017) used a high-fidelity CFD method to evaluate the modeling developed by Paryshev. CFD results were in consistent with the experimental data describing bulk cavity behavior, and showed that Paryshev's Model should be generalized to account for stagnation pressure losses of the jet. $\mathrm{Xu}$ Hao et al(2018) carried out an experimental study on the supersonic gaseous jet induced tail cavity at the wake of a revolution body, and investigated the transient evolution characteristics and forming mechanism. According to the observation, the induced cavities had four forms: foamy, intact, partially break, and pulsating foamy closure type.

Considering that enough propulsion is always required to insure that underwater vehicles could run steadily in a high speed, we prefer to concern the supercavtiy flows with a strong gas jet that could provide thrust no less than the cavitator $\operatorname{drag}(\bar{J}>1$, or $\bar{J} \approx 1)$. With sufficient momentum, such cavities could experience the instabilities of pulsation and collapse under certain circumstances. This article aims at investigating the instability of cavity with the strong jet impingement, and analyzing jet behavior in restricted space based on theoretical and numerical methods. Besides jet strength, the distance between the nozzle exit and cavity closure is considered as a very important parameter in jet/supercavity interaction. As the relative position varies, it will lead to diverse cavity surface evolution and different jet entrainment effect in cavity. In this paper, simulation for a supercavitating case without jet will be firstly carried out under different ventilation flow rate and comparison will be carried out with the experimental data to validate the numerical model. Then, by changing jet nozzle exit, a series of numerical simulation will be performed. The effect of the relative position on the jet/cavity interaction will be analyzed to provide useful methods in enhancing the stability of the ventilated cavity.

\section{Theoretical analysis of the jet/cavity interaction}

\subsection{Analysis of the key parameters}

Parametric analysis is a necessary task before numerical simulation. It shows all important parameters that may have an impact on the jet/cavity interaction including the jet parameter, the cavitation parameter and the geometry parameters. The transition criterion was bulit for the jet/cavity closure interaction mechanism by Paryshev and Money. Obviously, the parameter $\bar{J}$ and $\bar{P}$ represent implicitly the jet strength in the above criterions, but other parameters, like nozzle geometry and relative position, are not reflected in the discrimination mechanism. We have expanded on the basis of Paryshev's theory. Some CFD results are then shown which focused on the cavity flow field structure and physical process of cavity interface evolution, we try to perfect the theory analysis and find out the completed dimensionless parameters to describe the jet/cavity interaction, finally build criterions for the different interface evolution mechanism change.

First of all, the correlation between two non-dimensional numbers $\bar{P}, \bar{J}$ and the ratio of the nozzle exit area to the cavitator bottom area is derived. According to drag and mass flow rate formula equations, we could primitively get:

$$
\begin{gathered}
W_{c}=1 / 2 \rho_{\infty} U_{\infty} S_{D} C_{x} \\
\dot{m}_{j e t}=\rho_{j e t} U_{j e t} S_{j e t}
\end{gathered}
$$

In those relations, $S_{D}$ and $S_{j e t}$ denote the cone cavitator bottom area and the nozzle exit area respectively. In combination with Eqs.(6) and (7), furthermore, we could get:

$$
\bar{J} \cdot \bar{P}=\frac{2}{C_{x}} \frac{S_{j e t}}{S_{D}}
$$

The above relation shows that the effect of dimensionless nozzle exit area is reflected implicitly by the important parameter and $\bar{P}$. Despite all this, it could provide some meaningful advice for supercavity vehicle design. When thrust ( $\bar{J}$ ) 
is constant, we should improve $S_{j e t} / S_{D}$ to insure $\bar{P}>1$, so that the cavity is steady on basis of Paryshev's theory.

\subsection{Review of jet dynamic}

In order to understand the internal flow field structure of the cavity with a strong jet, we firstly review of gas jet dynamic knowledge. In general, the jets are classified in two types: free jet and confined jet (as shown in the figure 1 and 2 ) on the basis of the surrounding environment's effects. The free jet, often exists in an unlimited space and acts as a turbulent free jet. In this paper, we use a circle exit of the converging nozzle. Previous experimental research on the gas jet shows that, when the jet leaves from the orifice and enters into a quiescent environment, the velocity gradient results in the generation of turbulence eddies, the jet would entrains the surrounding gas and moves forward together, meanwhile, jet boundary spreads freely in the radial direction ${ }^{[16-19]}$. The whole jet development process is composed of two parts: the zone of flow establishment and the zone of established flow. Near the nozzle exit, a triangular area which is not affected by the entrainment and still maintain the original exit velocity is defined as the potential core. The regions between the potential core and the jet boundary is often called the shear layer (or mixing layer).The potential core length $\left(\mathrm{L}_{1}\right)$ depends on the geometry shape of convergent nozzle exit ${ }^{[20,21]}$.

$$
L_{1}=0.671 \frac{D_{j e t}}{2 a}
$$

Where $D_{j e t}$ is the diameter of the nozzle exit, and $a$ is the turbulence coefficient and could be obtained through experiment, it is often given as a range value of 0.053 0.071 for circle nozzle.

For a confined jet, a recirculation flow pattern is established because the amount of surrounding gas is not enough for the entrainment in presence of tank wall. So that the jet radius firstly increases and then decreases ${ }^{[23,24]}$. The jet boundary is featured and has experienced three stages: free expansion (section I to section II), restricted expansion (section II to section III), and contraction (section III to section IV). The presence of the walls will lead to the higher shear rates which increases the turbulence than that of free jet. Behind the recirculation region, the flow filed turns into the turbulent vortex region. The whole recirculation region length (section I to section IV) $\mathrm{L}_{2}$ is:

$$
L_{2}=3.58 \sqrt{A}+\frac{D_{j e t}}{a}\left(0.147 \frac{\sqrt{A}}{D_{j e t}}-0.133\right)
$$

Where A denotes the cross sectional area of the external tank wall.

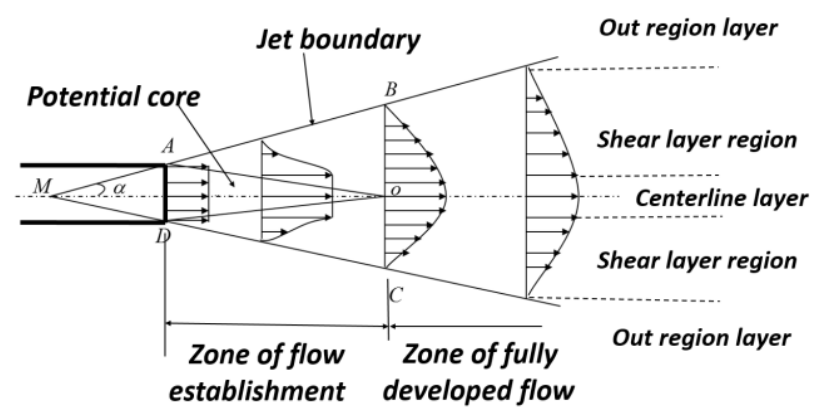

Fig. 1: Schematic of the turbulent round free jet.

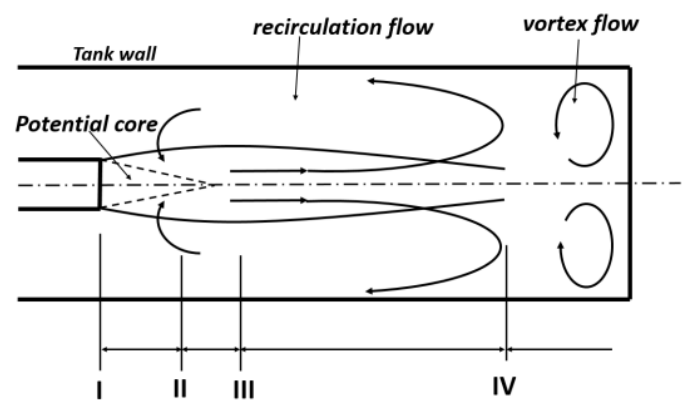

Fig. 2: Schematic of the turbulent round confined jet.

The above jet dynamic knowledge could help us to understand jet/surpercavity interaction. The jet structure in the cavity is similar to the confined jet, the only difference is that cavity interface is not fixed walls and relates to complex interface evolution. In one word, gas leakage ways induced by jet entrainment and cavity interface instabilities jointly determine the interface evolution mechanism. Under certain circumstances the jet entrainment would result in the atrophy of the cavity when the cavity don't have enough gas to maintain the cavity shape. On the one hand, the intensity of the entrainment effect is related to the jet strength. For an established supercavity, when jet momentum $\bar{J}$ is small, and the velocity gradient is low, the jet couldn't bring gas away from the cavity. As $\bar{J}$ increases to a certain value, due to the enhancement in velocity gradient shear, the jet starts to entrain surrounding gas and leave from the cavity together. The more gas that maintains the cavity are brought away, the more the cavity shrinks. As $\bar{J}$ continues to increase, the strengthened entrainment effect results in a pinch-off, pulsating, unsteady supercavity.

On the other hand, the strength of the entrainment effect is also related to the relative position. When the nozzle exit is nearer to the cavity closure, the shear layer (or mixing layer) zone of jet would diminish. It means that the surrounding gas entrained by jet decrease and may mitigate the negative effects caused by the jet. 


\subsection{Several situations of jet/cavity interaction}

For an established supercavity and a constant- $\bar{J}$ jet, the distance between the jet nozzle exit and the point of cavity closure $\left(S_{0}\right)$ is speculated to be an important parameter that impacts surface evolution mechanism for the cavity with a strong jet. However, in view of the asymmetric characteristics of the cavity surface at low Fr number, the jet would firstly interact with the lower cavity surface, rather than the closure region, so we redefine the distance parameter which represents the relative position of jet/cavity, that is the axis length of the nozzle outlet to the lower cavity surface. We also give the normalized form as

$$
\overline{S_{0}}=S_{0} / D_{n}
$$

When the relative position varies, several situations of jet/cavity interaction are analyzed as follows, Situation 1: the ventilated cavity closes on the slender body and has no connection with the tail cavity induced by jet. So we think that the jet has no effect on the cavity in theory, but in some water tunnel experiment, it is often noticed that the length of cavity is oscillating because of the test body vibrations or an uneven ventilation. That may lead to unexpected results in experiment, here, this is not our focus of concern.

Situation 2: when $0<S_{0}<L_{1}$ (or $0<\overline{S_{0}}<L_{1} / D_{n}$ ), the nozzle exit is nearer to the cavity closure and the cavity tail closes in the jet's potential core, the jet entrainment effect in cavity is very weak and almost negligible.

Situation3: when $L_{1}<S_{0}<L_{2}$ (or $L_{1} / D_{n}<S_{0}<L_{2} / D_{n}$ ), the cavity tail closes at the location outside the potential core, the jet entrainment effect enhanced.

Situation 4: when $S_{0}>L_{2}$ (or $S_{0}>L_{2} / D_{n}$ ), the cavity contains the entire jet flow, and the jet would entrain intensively the surrounding gas from the cavity. Presence of the strong entrainment at the large $\mathrm{S}_{0}$ will cause pressure and velocity pulsation in the cavity by, which could result in cavity interface evolution from a steady state to a new unsteady state.

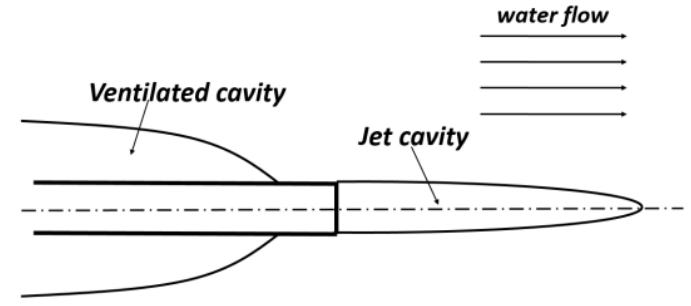

Situation1

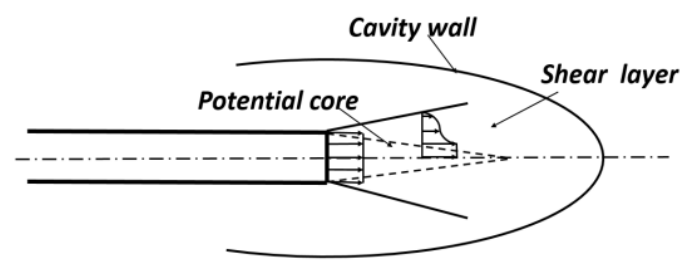

Situation 3

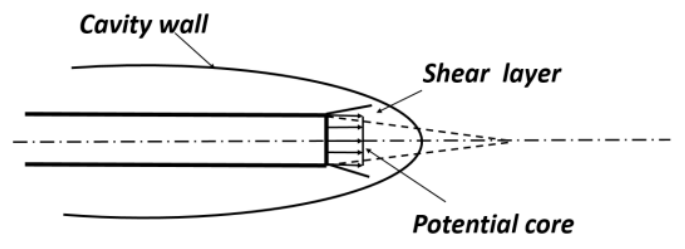

Situation 2

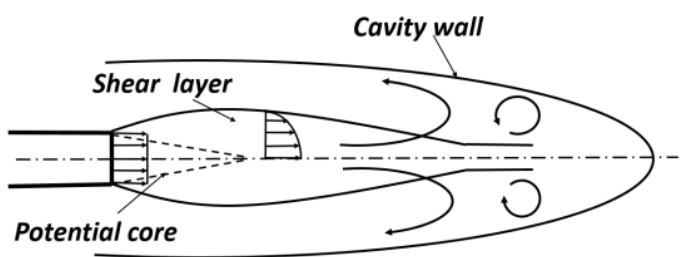

Situation 4

Fig. 3: The criterion for the jet/cavity interaction mechanism of varying relative position.

\section{Numerical Methods}

\subsection{Computational methodology}

In this manuscript, we use a homogeneous VOF multiphase model which is based on FLUENT fluid simulation platform. In the VOF model, a single set of momentum equations is shared by the fluids, and the fields for all material properties are shared by the phases and represent volume-averaged values, so long as the volume fraction of each phase is calculated at each control cell. There are two phase in the fluid field, an incompressible liquid phase for the external field and a incompressible non-condensable gas phase for the ventilation and jet. The liquid-gas phase interface is captured by a method which coupled VOF with level set. The governing equation:

$$
\begin{gathered}
\frac{\partial \rho}{\partial t}+\frac{\partial}{\partial x_{i}}\left(\rho u_{i}\right)=0 \\
\frac{\partial}{\partial t}\left(\rho u_{i}\right)+\frac{\partial}{\partial x_{j}}\left(\rho u_{i} u_{j}\right)=\frac{\partial p}{\partial x_{i}}+\frac{\partial}{\partial x_{j}}\left[\mu\left(\frac{\partial u_{i}}{\partial x_{j}}+\frac{\partial u_{j}}{\partial x_{i}}\right)\right]+\frac{\partial}{\partial x_{i}}\left(-\frac{2}{3} \mu \nabla \cdot V\right)
\end{gathered}
$$




$$
\frac{\partial \alpha_{l}}{\partial t}+\vec{v} \cdot \nabla \alpha_{l}=0
$$

The level-set function $\varphi(x, t)$ :

$$
\begin{aligned}
\varphi(x, t)= & \begin{cases}+|d|, & x \in \text { the primary phase } \\
0, & x \in \Gamma \\
-|d|, & x \in \text { the second phase }\end{cases} \\
& \frac{\partial \varphi}{\partial x}+\nabla \cdot(\mathbf{u} \varphi)=0
\end{aligned}
$$

\subsection{Physical Model, mesh and numerical condition description}

The object of simulation which used to research jet/cavity interaction is derived from Money's experimental model and some simplification is to be done to neglect the influence of the strut. The model consists of a 30 -degree, $31.75 \mathrm{~mm}$ diameter cone cavitator at the head of the slender body and a converging circular nozzle in the rear. The model has the geometrical characteristic of big cavitator, short body and small nozzle exit, the ratio of nozzle exit area to cavitator bottom area, $S_{j e t} / S_{D}$, is approximately equal to 0.12 . We know that it is easy to form unsteady supercavity flows with a strong gas jet from the previous analysis. In order to investigate the effect of relative position, we have designed four different length size for the physical model.

The symmetric surface of the computational domain is $3000 \mathrm{~mm} * 600 \mathrm{~mm}$. Grid refinement is carried out around the physical model body and in the nozzle tail domain. The number of mesh nodes is 1.7 million, which can better capture the interface variation of the cavity and the development of the jet morphology.

The boundary conditions are applied as follows: the velocity inlet and pressure outlet boundary conditions are used for the distribution of the incoming flow and outlet boundary, and the symmetric boundary condition is used for the symmetric surface. The non-slip wall boundary condition is used for the physical mode wall and the slip wall boundary condition is use for the far field. The ventilation and jet ports use mass flow inlet boundary.

\subsection{Verification and validation for a supercavitating case}

Firstly we have done a validation work of calculation methods for steady supercavity without jet by comparing the CFD results with theory results in the Fig.4 and 5. Guzevsky(1973) gave empirical formulas for the supercavity length, maximum diameter and drag coefficient of cone cavitator.

$$
\begin{gathered}
D_{c}=D_{n} \sqrt{\frac{C_{x}}{k \sigma_{c}}} \\
L_{c}=D_{n}\left[\frac{1.1}{\sigma_{c}}-\frac{4(1-2 \alpha)}{1+144 \alpha^{2}}\right] \sqrt{C_{x} \ln \frac{1}{\sigma_{c}}} \\
k=\frac{1+50 \sigma_{c}}{1+56.2 \sigma_{c}}
\end{gathered}
$$

Where $D c$ and $D n$ are the theoretical maximum diameter of the supercavity cross section and the diameter of the cone cavitator; $C x$ represents the drag coefficient of the cone cavitator.
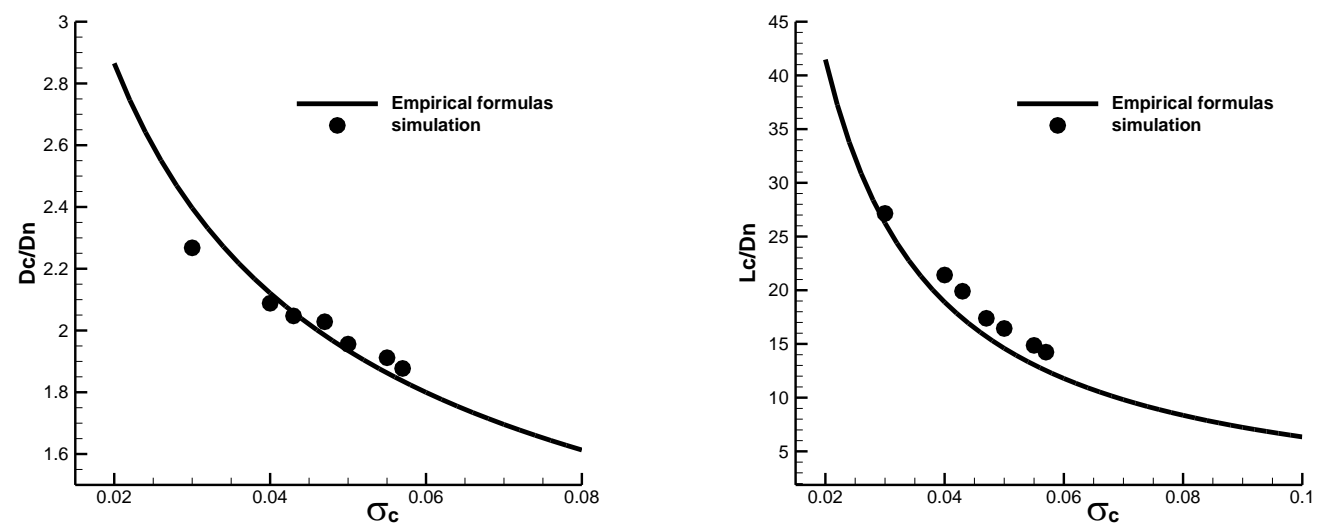

Fig. 4: The dimensionless maximum diameter and length of the cavity at different cavitation number. 


\section{Numerical results and discussions}

In order to study the effect of $\bar{S}_{0}$ on jet/supercavity interaction, one way is to change the ventilation rate or the free stream velocity so that the cavity closure region closes to the nozzle exit, the other way is to elongate the model's body to make that the nozzle exit closes to the cavity closure region. We choose the latter because that could avoid introducing other variables when different $\bar{S}_{0}$ is analyzed. We firstly simulate a steady cavity in the condition of $F r=11.4, C q=0.16$. And then a strong jet with strength of $\bar{J}=1, \vec{P}=1.355$ and $\bar{J}=1.88, \bar{P}=0.72$ is generated. Thereafter, by changing the relative position of nozzle exit to the cavity with four different length of physical models, a series of numerical simulations of supercavitation flows is performed.

According to formula equation (9), the dimensionless jet potential core length is obtained:

$$
L_{1} / D_{n}=\frac{0.671}{2 a} \frac{D_{\text {jet }}}{D_{n}}
$$

According to formula equation (10) and (21), where A , the cross sectional area in the confined space is decided by the maximum cross sectional area of the cavity, the dimensionless recirculation region length of the jet is obtained:

$$
L_{2} / D_{n}=\left(3.58+\frac{0.147}{a}\right) \sqrt{\frac{\pi}{8}} \frac{D_{C}}{D_{n}}-\frac{0.133}{a} \frac{D_{\text {jet }}}{D_{n}}
$$

The theoretical value of $L_{1} / D_{n}$ and $L_{2} / D_{n}$ are 2.19 and 6.4 respectively. Fig.5 shows four groups of the relative position between nozzle exit and cavity. Combined with the discriminant criterion for the jet/cavity interaction mechanism of varying relative position, these calculation cases could be reasonably classified: the case $1 \mathrm{a}$, case $1 \mathrm{~b}$, case $2 \mathrm{a}$ and case $2 \mathrm{~b}$ belong to situation 4 , the case $3 \mathrm{a}$ and case $3 \mathrm{~b}$ belong to situation 3 , and the case $4 \mathrm{a}$ and case $4 \mathrm{~b}$ belong to situation 2 .
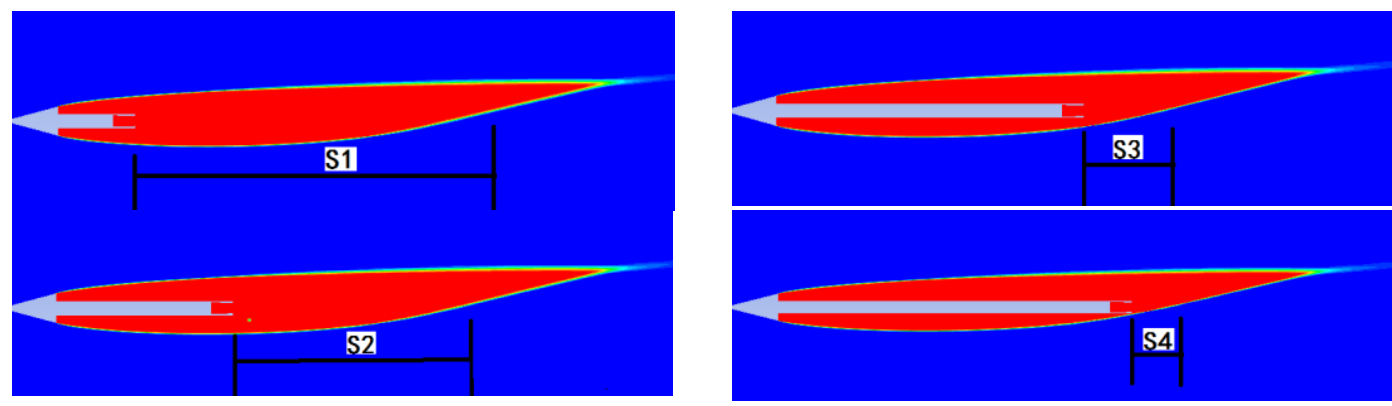

Fig. 5: The relative position between nozzle exit and cavity.

\subsection{The cavity interface transient evolution process}

Fig. 6 and fig. 7 show respectively the cavity interface transient evolution process in the case $1 \mathrm{~b}$ and case $4 \mathrm{~b}$. As for the case1b $\left(\bar{P}<1\right.$ and $\left.S_{0}>L_{2} / D_{n}\right)$, a comparison is showed between CFD predictions of the cavity interface evolution with a strong jet and Money's experiment results. The numerical methods are proved appropriate in capturing interface motion for the cavity. It can be clearly seen from the CFD results that the cavity bubble break off and shed due to elongated morphology, while in the experiment, because of the rapid instability of gas-liquid interface and the mixing of water and gas, a large number of continuous broken bubbles are actually observed.

The cavity interface evolution process could be subdivided into two stages according to present simulation and experiment dates. The first stage, the cavity changes through an expansion, necking, shedding process with jet effects. The total cavity get a reduction in volume by retraction, which is showed as the elastic behavior of cavity interface. Then, the second stage, the cavity presents periodic gas-leakage in the tail and maintain this unsteady state of affairs continued. But for case $4 \mathrm{~b}\left(\vec{P}<1\right.$ and $\left.0<S_{0}<L_{1} / D_{n}\right)$, the first stage is not obvious, after a period of temporary the cavity begins directly to leak gas in the way of the periodic pulsation pattern.

In the cavity interface evolution process, the states of two moments are emphatically analyzed. The moment of $t=0.15 \mathrm{~s}$ is that the final shape of the cavity forms in the periodic varying state of growth and shedding for dynamic equilibrium. And the moment of $t=0.005 \mathrm{~s}$ is that the jet has just formed and the cavity shape has not changed yet. It is meaningful to study the evolution mechanism of the cavity interface, which analyze the structural characteristics of the confined jet, the strength of the entrainment effect and it's influence on the quality change in the cavity at this moment.

Fig. 8 shows the cavity morphology characteristics for varying relative position and jet strength at $\mathrm{T}=0.15 \mathrm{~s}$. It is summarized from the observation of different cavity interface evolution process, when $\bar{P}>1$, the periodic gas-leakage phenomenon is not obvious, the cavity deflates in pattern of axial flow (Efros-type) so that the cavity tail looks slender; when $\bar{P}<1$, the periodic pulsation gas-leakage phenomenon in the tail is very obvious. As for the influence of the relative position, the cavity interface evolution's first stage is very obvious for case S1 and case S2, but for case S3 and case S4, it turns to be not obvious. As the distance parameter $\mathrm{S}_{0}$ decreases, the cavity volume showed a trend of increase. Compared to 
the cavity without jet, the result of case S1 and case S2 are much smaller, but the result of case S3 and case S4 tend to be slightly larger. From the view of the cavity instability with internal jet perturbations, the case S1_ $\bar{J}=1.88$ is the most unstable, which has distinct interface fluctuation, jet perturbations is the weakest in the case $S_{-} \bar{J}=1$, which has the most stable state.In the process of unsteady gas-leakage, the cavity would present periodic expansion and shedding. When the cavity varies in the process of expansion, the pressure would decrease. At the moment of shedding, the pressure changes very fast that makes the pressure cure behaves as a broken line. When $\bar{P}>1$, the pressure fluctuation is small because the cavity is relatively stable in the axial flow gas-leakage pattern; when $\bar{P}<1$, the pressure fluctuation is very strong because the cavity is unstable in the periodic pulsation pattern.

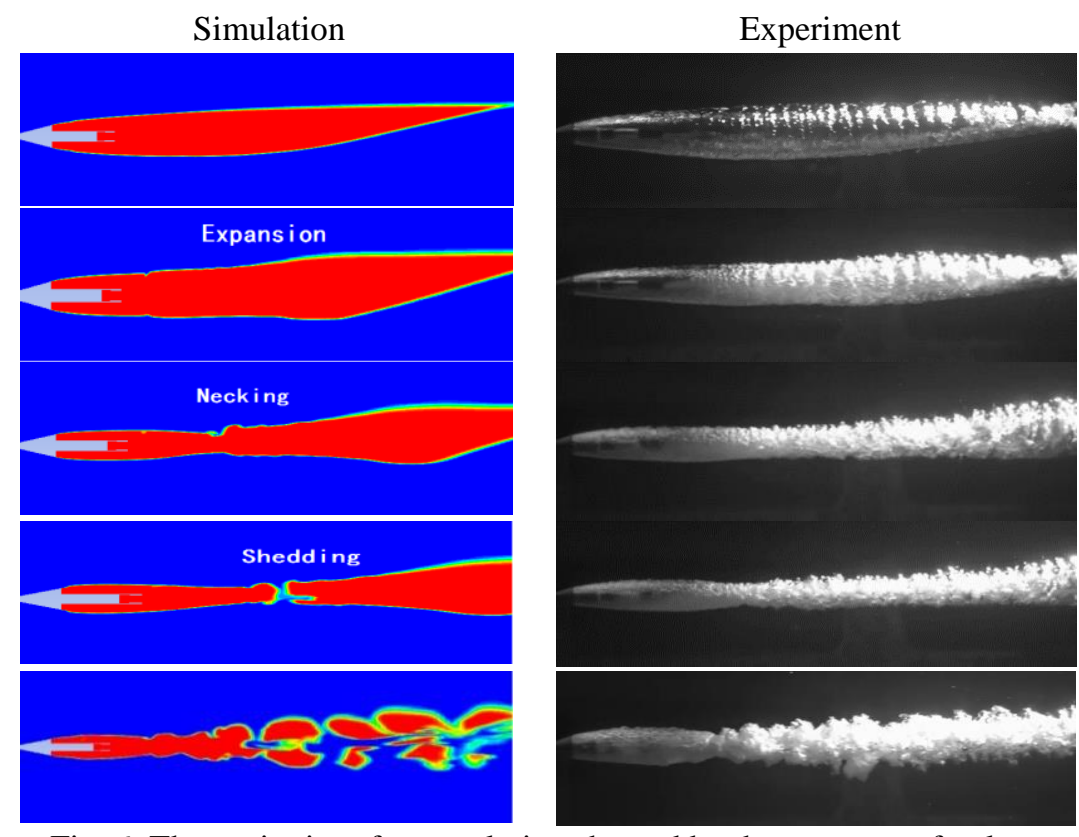

Fig. 6: The cavity interface evolution showed by the contours of volume fraction when $\bar{P}<1$ and $\overline{S_{0}}>L_{2} / D_{n}$ (blue regions: water, red regions: gas).

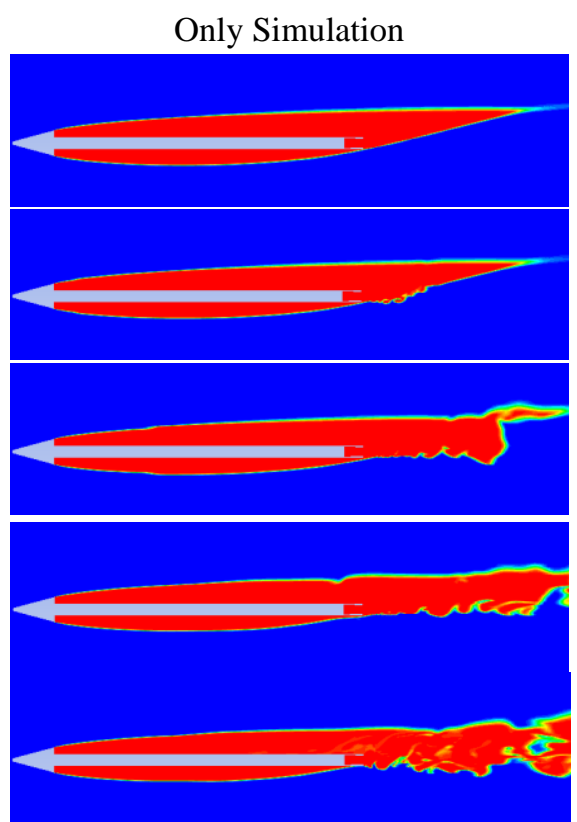

Fig. 7: The cavity interface evolution when $\bar{P}<1$ and $0<\overline{S_{0}}<L_{1} / D_{n}$
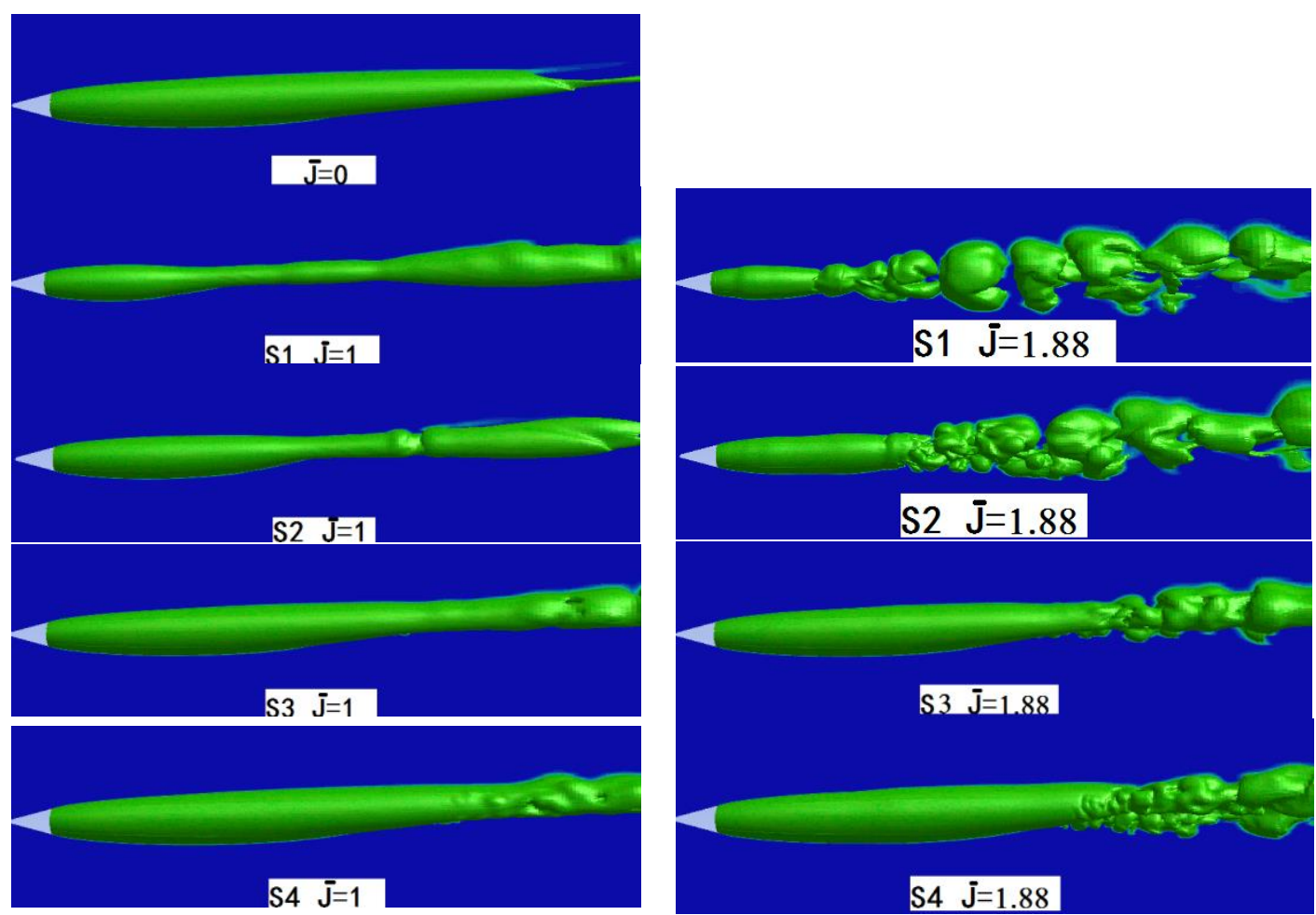

Fig. 8: The cavity morphology characteristics for varying relative position and jet strength at $\mathrm{T}=0.15 \mathrm{~s}$. The cavity interface is obtained by gas volume fraction of 0.5 . 


\subsection{The confined jet structure in a variable cavity}

The jet spreads inside the cavity, which will result the fluctuation and deformation of the cavity surface. On the other side, the change of the cavity will also give rise to variation of the jet style due to the change of the ambient flow parameters. Fig.9 shows velocity streamline diagram in the cavity flow field with a gas jet as the distance parameter varies. Before the jet was generated, the gas near the gas-liquid interface is dragged by the water flow, the internal cavity appears backflow in the opposite direction. After the jet was generated, the internal cavity flow is completely dominated by the jet and vortex direction is changed. In case $\mathrm{S} 1$, due to asymmetrical entrainment effect by the jet, the cavity has asymmetrical vortex structure, that gas dragged by the external water flow and re-entrant jet conspire to form a small vortex in front. In case S2, the vortex in the lower cavity almost disappears. In case S3 and case S3, vortex structure is obviously limited by the narrow space. In particular, instead of a big vortex, it becomes vortex pair is in case S4.
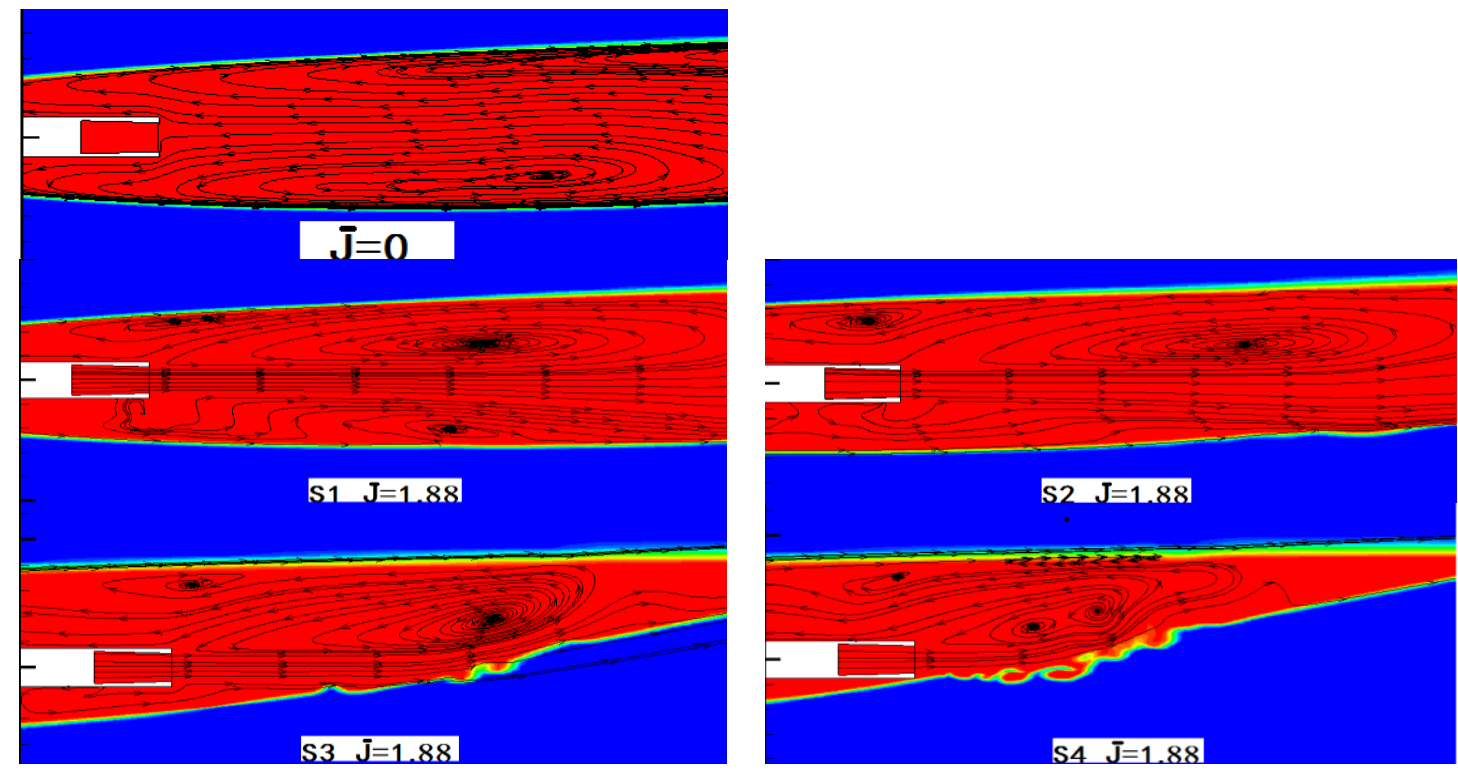

Fig. 9: As the relative position varies, the velocity streamline in the cavity is showed at $\mathrm{T}=0.005 \mathrm{~s}$
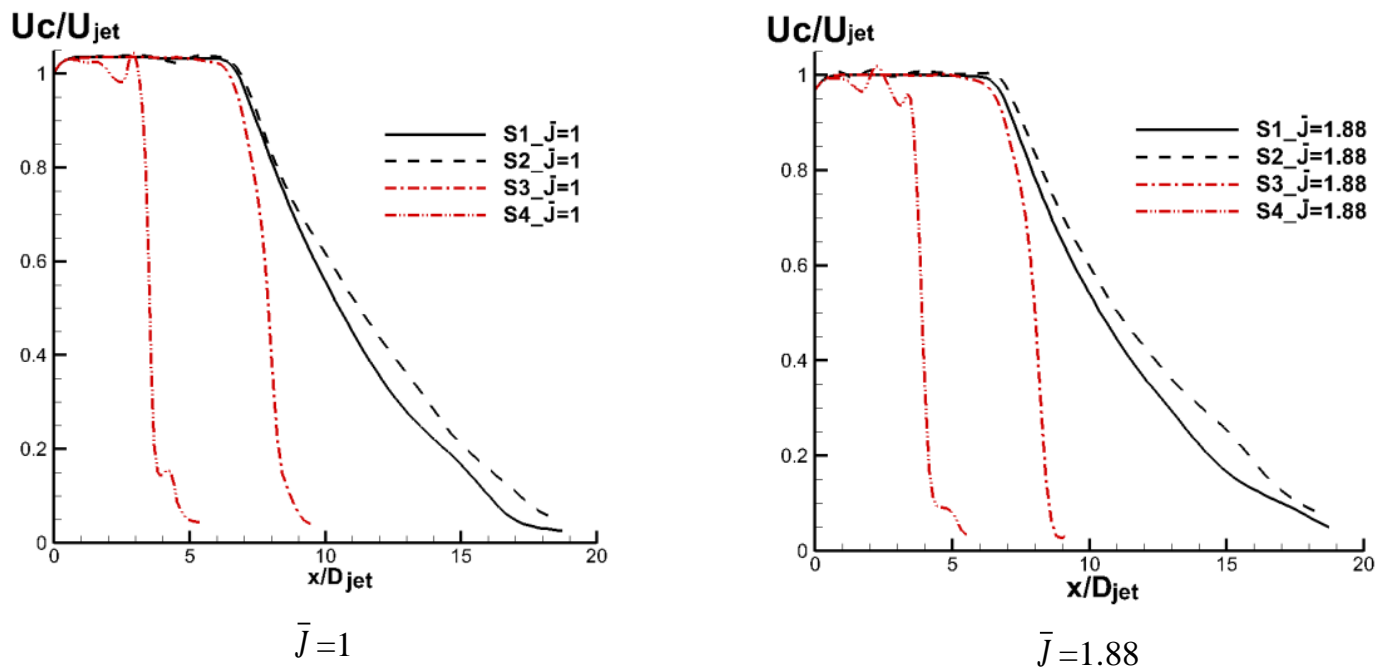

Fig. 10: The normalized centerline axial mean velocity decay at $\mathrm{T}=0.005 \mathrm{~s}$.

Fig.10 shows the normalized centerline axial mean velocity decay of the jet for varying relative position and jet strength at $\mathrm{T}=0.005 \mathrm{~s}$. As for case $\mathrm{S} 1$ and case $\mathrm{S} 2$, jet structure is a symmetrical and fully developed type, which is weakly disturbed from the under cavity wall. By considering momentum balance, the entrainment effect intensity of case S1 is stronger than that of case S2, so the centerline axial mean velocity decay faster in case S1 which compared with case S2. But for case S3 and case S4, jet structure is an asymmetrical and developing type, which appears across the media and encounters with the stagnation effect from external water fluid, so the centerline axial mean velocity decay very fast. 


\subsection{Quantitative analysis of the entrainment effect}

Fig.11 shows the cavity internal flow structure with a gas jet. Jet entrainment effect results in a recirculation flow in the cavity interior and build a new gas-leakage pattern in the cavity tail. The entrainment mass flow could been obtained by the mass flow at the jet cross section along the axial direction minus the mass flow at the nozzle exit. Fig. 12 shows the normalized entrainment rates of the surrounding gas into the jet for varying relative position and jet strength at $\mathrm{T}=0.005 \mathrm{~s}$. Along the axial direction, the entrainment rate firstly increases and then decreases. For all cases, a peak in the entrainment rate cures could be observed and the maximum entrainment rate appears at the maximum radius of the jet. It can be seen that the stronger the jet is, the larger the entrainment rates are, and the nearer relative position $\overline{S_{0}}$ is, the smaller the entrainment rates are. The non-dimensional parameter $\bar{J}$ and $S_{0}$ jointly determine the entrainment effect intensity.

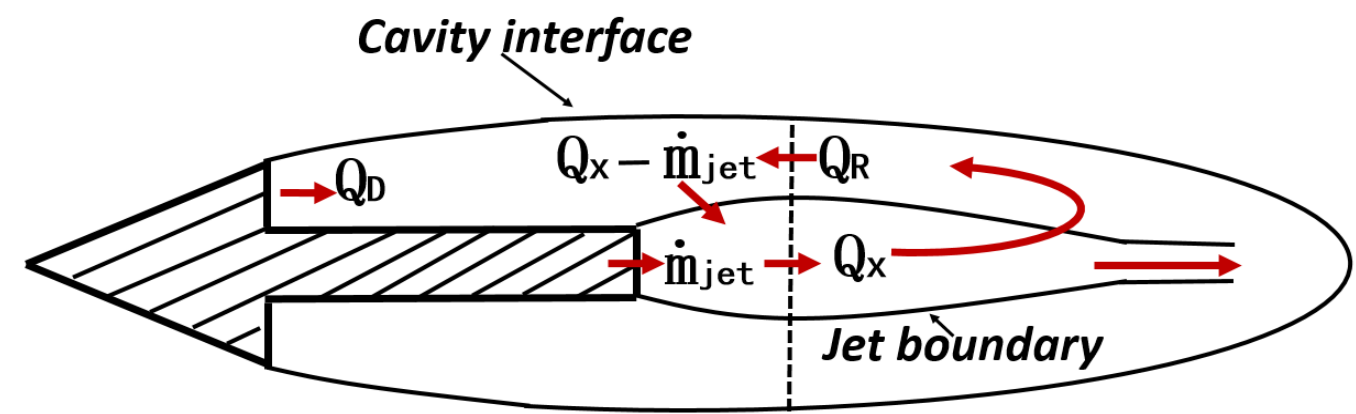

Fig. 11: Schematic of the cavity internal flow structure with a gas jet.

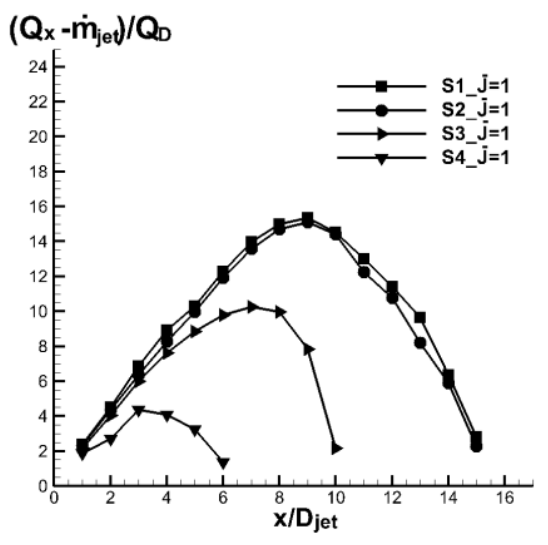

$\bar{J}=1$

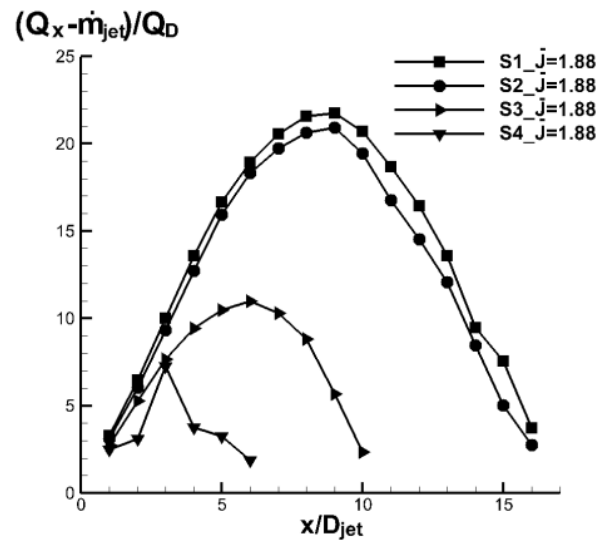

$\bar{J}=1.88$

Fig. 12: The normalized entrainment rates of the surrounding gas into the jet along the axial direction at $\mathrm{T}=0.005 \mathrm{~s}$. Qx denotes the mass flow rate across the jet section. $\dot{m}_{j e t}$ and $\mathrm{Q}_{\mathrm{D}}$ denotes the mass flow rate of the jet and ventilation. 
Table 1: The entrainment and recirculation mass flow rate for each case at $T=0.005 \mathrm{~s}$. The max entrainment mass flow rate is obtained at the max jet radius. The dates in the table are half of the actual value for one half of computational domain.

\begin{tabular}{cccccc}
\hline \hline Case & $\begin{array}{c}\text { Ventilation } \\
\text { flow rate } \\
Q_{D}(\mathbf{k g} / \mathbf{s})\end{array}$ & $\begin{array}{c}\text { Jet flow } \\
\text { rate } \\
\dot{m}_{\text {jet }}(\mathbf{k g} / \mathbf{s})\end{array}$ & $\begin{array}{c}\text { The maximum } \\
\text { entrainment } \\
\text { flow rate } \\
Q_{x}-\dot{m}_{\text {jet }}(\mathbf{k g} / \mathbf{s})\end{array}$ & $\begin{array}{c}\text { Recirculation } \\
\text { flow rate } \\
Q_{R}(\mathbf{k g} / \mathbf{s})\end{array}$ & $\begin{array}{c}Q_{x}-\dot{m}_{\text {jet }} \\
-Q_{D}-Q_{R}\end{array}$ \\
\hline \hline $\mathrm{S} 1 \_\bar{J}=1$ & 0.00062328 & 0.00908307 & 0.00956197 & 0.0088959 & $>0$ \\
$\mathrm{~S} 2 \_\bar{J}=1$ & 0.00062328 & 0.00908307 & 0.00941998 & 0.0086789 & $>0$ \\
$\mathrm{~S} 3 \_\bar{J}=1$ & 0.00062328 & 0.00908307 & 0.00638417 & 0.0079659 & $<0$ \\
$\mathrm{~S} 4{ }_{-} \bar{J}=1$ & 0.00062328 & 0.00908307 & 0.00273304 & 0.0045968 & $<0$ \\
$\mathrm{~S} 1 \_\bar{J}=1.88$ & 0.00062328 & 0.0124656 & 0.0135627 & 0.0123124 & $>0$ \\
$\mathrm{~S} 2 \_\bar{J}=1.88$ & 0.00062328 & 0.0124656 & 0.0133854 & 0.0114708 & $>0$ \\
$\mathrm{~S} 3 \_\bar{J}=1.88$ & 0.00062328 & 0.0124656 & 0.0068512 & 0.0084827 & $<0$ \\
$\mathrm{~S} 4{ }_{-} \bar{J}=1.88$ & 0.00062328 & 0.0124656 & 0.0045186 & 0.0067525 & $<0$ \\
\hline
\end{tabular}

Table 4 shows the entrainment and recirculation mass flow rate for each case at $\mathrm{T}=0.005 \mathrm{~s}$. So that we could make a quantitative analysis to indicate the morphological changes of the cavity. By calculating the max entrainment flow rate plus ventilation and recirculation flow rate, we could judge whether the cavity is smaller at the next time step. When $Q_{x}-\dot{m}_{\text {jet }}-Q_{D}-Q_{R}>0$, it deflates the cavity so that the cavity will turns smaller; when $Q_{x}-\dot{m}_{\text {jet }}-Q_{D}-Q_{R}<0$, it inflates the cavity so that the cavity will turns slight larger.

The cavity interface evolution process are also the rebuilding process from an unbalance state to a dynamic balance state, in which the beginning is $Q_{x}-\dot{m}_{\text {jet }}-Q_{D}-Q_{R}>0$ or $<0$, and the ending is $Q_{x}-\dot{m}_{\text {jet }}-Q_{D}-Q_{R} \approx 0$ for $\bar{P}>1$ or the arithmetic mean value in one period approximately equal to zero for $\bar{P}<1$.

\section{Conclusions}

Numerical simulation is extremely important for the understanding of jet/cavity interaction. In this paper, a multiphase model using coupled VOF and level set method is adopted to capture gas-liquid interface. The cavity interface evolution process have been studied and summarized based on present simulation and experiment dates. Then, the criterion is analyzed and built for the different jet/cavity interaction mechanism of varying jet strength and relative position.

A series of numerical simulations of supercavitation flows was performed to verify the analysis of jet/cavity interaction for varying relative position. Numerical results show a good agreement to support our predictions. A non-dimensional distance parameter is proposed to define the interaction schemes between jet and cavity. The non-dimensional parameter $\overline{\boldsymbol{J}}$ and $\bar{S}_{0}$ jointly determine the entrainment effect intensity.

Finally, a quantitative analysis method has been studied, which is to associate entrainment effect and the cavity interface evolution. In future work, we will try to construct semi-empirical formulas between cavity dimensions and $\bar{J}, \overline{S_{0}}, \mathrm{Fr}, \mathrm{Cq}$ etc. It should be an important and practical theory in the artificial supercavition technique.Acknowledgments

\section{Acknowledgments}

The authors gratefully acknowledge support by the National Nature Science Foundation of China (NSFC, Grant NO: $51776221)$.

\section{References}

[1] Reichardt $\mathrm{H}$. The laws if cavitation bubbles as axially symmetrical bodies in a flow. Britian : Ministry of Aircraft Production Reports and Translations, 1946.

[2] Garabedian P R. Calculation of axially symmetric cavities and jets. Pacific Journal of Mathematics. 1956 , vol. 6, no. 4, pp. 611-684.

[3] Cox R N, Clayden W A. Air Entrainment at the Rear of a Steady Cavity. Proceedings of the N.P.L. Symposium on Cavitation in Hydrodynamics, London, 1956.

[4] Waid R L. Cavity Shapes for Circular Disks at Angles of Attack. California Institute of Technology. Report No.E-73.4, 1957.

[5] Campbell I J, Hilborne D V. Air Entrainment Behind Artificially Inflated Cavities. Second Symposium on Cavitation on Naval Hydrodynamics, Washington, 1958.

[6] Silberman E, Song C S. Instability of Ventilated Cavities. St. Anthony Falls Hydraulic Laboratory, University of Minnesota. Technical Paper No.29, Series B,1959. 
[7] Logvinovich G V. Hydrodynamics of flows with free boundaries. Naukova Dumka Publishing, Kiev, 1969(in Russian).

[8] Epshtein L A. Characteristics of ventilated cavities and some scale effects.Proc. of Int. Symposium IUTAM, Nauka Publishing House, Moscow,1973.

[9] Semenenko V N. Artificial supercavitation: physics and calculation. RTO AVT Lecture Series on Supercavitating Flows, Von Karman Institute, Brussels, Belgium, 2001.

[10] Paryshev E V. Approximate Mathematical Models in High-Speed Hydrodynamics. Journal of Engineering Mathematics. 2006,55(1-4), pp. 41-64.

[11] Moeny M J, Krane M H, Kirschner I N, Kinzel M P. Jet-Supercavity Interaction: Insights from experiments. In: Proceedings of the 9th International Symposium on Cavitation (CAV2015).2015.

[12] Kinzel M P, Krane M H, Kirschner I N, Moeny M J. A numerical assessment of the interaction of a supercavitating flow with a gas jet.Ocean Engineerin.2017,136, pp. 304-313.

[13] Bourlioux A. A coupled level-set volume-of-fluid method for tracking material interfaces. In: Proceedings 6th Annual Int. Symp.on Comp. Fluid Dynamics, Lake Tahoe, USA.1995.

[14] Sussman M, Puckett E G. A coupled level set and volume-of-fluid method for computing 3D and axisymmetric incompressible two-phase flows. J.Comput.Phys. 162(2000)301-337.

[15] Albertson M L, Dai Y B, Jensen R A et al. Diffusion of submerged jets. Transactions ASCE. 1950,115, pp. 639-664.

[16] Schwarz W H. The radial free jet. Chemical Engineering Science.1963,Vol.18, pp.779-786.

[17] Ball C G, Fellouah H, Pollard A. The flow field in turbulent round free jets. Progress in Aerospace Sciences. 50(2012) pp. 1-26.

[18] Rao S M V, Asano S, Saito T. Comparative studies on supersonic free jets from nozzles of complex geometry. Applied Thermal Engineering. 99(2016) pp. 599-612.

[19] Sadeghi H, Lavoie P, Pollard A. Scale-by-scale budget equation and its self-preservation in the shear-layer of a free round jet. International Journal of Heat and Fluid Flow. 61(2016) pp. 85-95.

[20] Kandakure M T, Patkar V C, Patwardhan A W. Characteristics of turbulent confined jets. Chemical Engineering and Processing. 47(2008) pp. 1234-1245.

[21] Rahman M S, Tay G F K, Essel E E, Tachie M F. Effects of offset height on the turbulent characteristics of a surface attaching jet. International Journal of Heat and Fluid Flow.71 (2018) pp. 305-321. 